NBER WORKING PAPER SERIES

\title{
CONVERGING TO CONVERGE? A COMMENT
}

\author{
Daron Acemoglu \\ Carlos A. Molina \\ Working Paper 28992 \\ http://www.nber.org/papers/w28992
NATIONAL BUREAU OF ECONOMIC RESEARCH
1050 Massachusetts Avenue
Cambridge, MA 02138
July 2021

This paper is an expanded version of the commentary presented at the 2021 NBER Macroeconomics Annual conference, which will be published in that volume. We gratefully acknowledge financial support from the Bradley Foundation. The views expressed herein are those of the authors and do not necessarily reflect the views of the National Bureau of Economic Research.

NBER working papers are circulated for discussion and comment purposes. They have not been peer-reviewed or been subject to the review by the NBER Board of Directors that accompanies official NBER publications.

(C) 2021 by Daron Acemoglu and Carlos A. Molina. All rights reserved. Short sections of text, not to exceed two paragraphs, may be quoted without explicit permission provided that full credit, including (C) notice, is given to the source. 
Converging to Converge? A Comment

Daron Acemoglu and Carlos A. Molina

NBER Working Paper No. 28992

July 2021

JEL No. O47,P16

\begin{abstract}
Kremer, Willis, and You (2021) revisit cross-country convergence patterns over the last six decades. They provide evidence that the lack of convergence that applied early in the sample has now been replaced by modest convergence. They also argue this relationship is driven by convergence in various determinants of economic growth across countries and a flattening of the relationship between these determinants and growth. Although the patterns documented by the authors are intriguing, our reanalysis finds that these results are driven by the lack of country fixed effects controlling for unobserved determinants of GDP per capita across countries. We show theoretically and empirically that failure to include country fixed effects will create a bias in convergence coeffcients towards zero and this bias can be time-varying, even when the underlying country-level parameters are stable. These results are relevant not just for the current paper, but for the convergence literature more generally. Our reanalysis finds no evidence of major changes in patterns of convergence and, more importantly, no flattening of the relationship between institutional variables and economic growth. Focusing on democracy, we show that this variable's impact continues to be precisely estimated and if anything a little larger than at the beginning of the sample.
\end{abstract}

Daron Acemoglu

Department of Economics, E52-446

Massachusetts Institute of Technology

77 Massachusetts Avenue

Cambridge, MA 02139

and NBER

daron@mit.edu

Carlos A. Molina

Massachusetts Institute of Technology

77 Massachusetts Ave

Cambridge, MA 02139

camolina@mit.edu 


\section{Introduction}

Kremer et al. (2021) revisit how the global distribution of prosperity and growth have evolved over the last six decades and the role of various factors in shaping these distributions. Building on the convergence framework of Barro (1991) and Barro and Sala-i Martin (1992), they investigate how a country's GDP per capita today depends on its GDP per capita in the past (unconditional convergence) and whether this relationship is different when conditioning on various determinants or "correlates" of growth (conditional convergence). While the earlier literature concluded that there was unconditional divergence and conditional convergence, Kremer et al. report a trend towards unconditional convergence (meaning that growth in rich countries is no longer faster, and in fact may be slower than, in poor countries). They also find that this trend towards convergence has been accompanied by rapid convergence among the correlates of income - in particular human capital, policies, institutions and culture. Finally, they report results suggesting that the relationship between growth and these correlates is now flatter, which they interpret as these factors becoming less important for economic growth, perhaps because remaining differences between institutions and policies are more minor or are confined to areas that matter less. Although the authors are careful in not pushing a very strong interpretation of this last finding, some may read these findings as suggesting that improving institutions and policies may have become less important in the modern era.

There is no doubt that these are first-order questions for economic growth and development, and the authors' voice and novel analysis are welcome additions to this debate. Their paper documents intriguing and thought-provoking facts. However, our assessment is that their findings suffer from some of the shortcomings that are inherent in the convergence framework. We take this opportunity both to comment on Kremer et al. and the broader convergence literature pioneered by Barro and Sala-i-Martin.

We argue that unconditional convergence regressions, especially in the form formulated by the authors, do not allow a straightforward causal interpretation. Lack of convergence may be because countries differ in their institutions and policies. It may be because of technological divergence due to other reasons. Or it may be because of statistical problems, in particular when the framework at hand does not properly account for permanent differences rooted in other factors. These shortcomings do not just make it difficult to interpret what estimates of unconditional convergence/divergence mean, but also imply that changes in convergence patterns may be a statistical artifact of the same problems as well.

To elucidate these issues, we start with a framework that is more amenable to thinking and estimating causal effects - specifically, by allowing heterogeneity across countries, for example, as captured by country fixed effects. Using this framework, we first establish that convergence estimates do not have a straightforward economic interpretation. For example, even if every country had a negative converges coefficient (indicating convergence), the authors' estimates 
could be strongly positive. This bias is not only first-order but it is potentially time-varying and can create the impression that convergence patterns are changing, when, in reality, it is the statistical properties responsible for the bias that are evolving over time.

We then reanalyze the same data as the authors and report several important findings $\mathrm{H}^{1}$ First, in the data, there is indeed a major discord between estimated convergence coefficients (without fixed effects) and economically-meaningful parameters summarizing how growth depends on current level of income. For example, we estimate that more than $88 \%$ of all countries show evidence of convergence, while the authors' regressions for the whole sample show no convergence. Second, the biases responsible for this discord are indeed time-varying and account for the changes in convergence patterns the authors report. Third, this framework also enables us to estimate the effects of key institutional factors and policies on growth. Carrying out these estimates for one specific institutional characteristic - whether a country is a democracy — we find very different results from those reported by Kremer et al. Specifically, we confirm the results in Acemoglu, Naidu, Restrepo, and Robinson (2019), showing that democracy has a statistically robust and economically large positive effect on GDP per capita. Moreover, contrary to the findings of Kremer et al., there is no evidence of the relationship between democracy and growth flattening over time. This suggests there should be no presumption that institutional factors in general have become less important for explaining and spearheading economic growth.

The rest of the paper is organized as follows. In the next section, we start with a brief review of a minimalist framework that can shed light on the causal relationship between different factors and GDP per capita (or growth). We contrast this framework, which crucially includes country fixed effects to control for unobserved or unmodeled country-level determinants of GDP per capita, with the authors' model which omits these fixed effects. In Section 3, we theoretically and empirically explore the implications of our framework, establishing that the failure to include country fixed effects will lead to a (potentially time-varying) downward bias in estimates of convergence. We document that this bias explains both the lack of convergence estimated in the earlier literature (and by the authors in the full sample) and the pattern towards greater convergence over time. In Section 4, we examine whether the relationship between economic growth and its potential determinants (and especially democracy) has become weaker over time, and we find no evidence that it has. In Section 5, we respond to the adjustments that the authors have made following our conference comments, while Section 6 concludes. The Appendix, which is available online, includes the proof of our theoretical result and some additional empirical findings.

\footnotetext{
${ }^{1}$ Namely, we follow Kremer et al. ss first draft, on which our comments were based, in using GDP per capita data from the World Development Indicators database. Their final draft switches to GDP numbers from the Penn World Tables. We use the former dataset in the text and repeat all of the same exercises with the Penn World Tables in the Appendix to verify that the choice of dataset does not matter for any of the points we emphasize.
} 


\section{$2 \quad$ A Minimalist Framework}

Consider the following relationship linking a country's economic growth to various characteristics, including its current level of GDP per capita:

$$
\Delta y_{c t}=\beta_{c} x_{c t}+\rho_{c} y_{c t-1}+\delta_{t}+\alpha_{c}+\varepsilon_{c t},
$$

where $y_{c t}$ is the level of log GDP per capita of country $c$ at time $t$ and $x_{c t}$ is some institutional/policy feature potentially impacting economic growth. In addition, $\alpha_{c}$ stands for country fixed effects, which capture various dimensions of country heterogeneity that influence economic growth. These may include other institutional features or various unobserved and/or unmodeled factors. Finally, $\varepsilon_{c t}$ is an error term and $\delta_{t}$ denotes time effects. This relationship allows for heterogeneities, including in how institutional/policy features impact growth $\left(\beta_{c}\right)$ and the extent of persistence $\left(\rho_{c}\right)$, but is assumed to be linear for simplicity.

In what follows, we assume that equation (1) is the true model/data generating process (DGP), and also assume that $x_{c t}$ is orthogonal to $\varepsilon_{c t}$ (conditional on $x_{c t}$ and $\alpha_{c}$ ).

Equation (1) is similar to the conditional convergence framework of Barro (1991) and Barro and Sala-i Martin (1992), because the relationship between $\Delta y_{c t}$ and $y_{c t-1}$ is conditional on $x_{c t}$ and $\alpha_{c}$. However, these papers do not typically include country fixed effects capturing unobserved permanent differences across countries, which will prove to be important.2

Now consider a typical unconditional convergence equation:

$$
\Delta y_{c t}=\rho y_{c t-1}+\delta_{t}+\varepsilon_{c t}
$$

This equation is a special case of (1), with three differences. First and most importantly, the $\alpha_{c}$ term is not present, implying that the model does not allow for (unobserved) heterogeneity across countries that could be correlated with initial GDP per capita. Second, there is no $x_{c t}$, which could capture some of the observed differences across countries. Third, this equation does not allow for any cross-country heterogeneity in the relationship between GDP per capita and its growth (captured by $\rho_{c}$ in (1)). Kremer et al. use this equation to estimate $\rho$ and study unconditional convergence, though they look at the 10-year change, $\Delta_{10} y_{c t}=y_{c t+10}-y_{c t}$, on the left-hand side, rather than the annual change as in (2). We next show that the omission of appropriate country heterogeneity in equation (2) makes their estimates of $\rho$ difficult to interpret.

\footnotetext{
${ }^{2}$ Other papers that have explored this type of linear model with country fixed effects include Knight, Loayza, and Villanueva (1993); Loayza (1994); Islam (1995); Caselli, Esquivel, and Lefort (1996) as well as Acemoglu et al. (2019).
} 


\section{Implications of Country Heterogeneity}

Let us now suppose that the DGP is given by our equation (1). What happens when $\rho$ is estimated from equation (2)? We answer this question first in theory, and then in the context of the authors' analysis of convergence.

\subsection{Theory}

In order to separate the implications of country heterogeneity from those of country covariates which Kremer et al. later incorporate, we first set $\beta_{c}=0$ for all $c$ in equation (1). This ensures that the only difference between the underlying causal model, (1), and the authors' statistical model, (2), is the absence of country fixed effects.

Proposition 1 Suppose the DGP is (1) with $\beta_{c}=0$ for all c. Let $\hat{\rho}$ be the OLS estimator of $\rho$ in equation (2). Then:

1. plim $\hat{\rho}=\sum_{c=1}^{C} \omega_{c} \rho_{c}$. Where $\omega_{c}^{\prime}$ 's can be negative or greater than one, and $\sum_{c=1}^{C} \omega_{c}$ is typically not equal to one.

2. Let $\theta_{c}=\omega_{c}-\frac{\theta_{0} k}{C \rho_{c}}$, where $\theta_{0}=1-\sum_{c=1}^{C} \theta_{c}, \theta_{c} \in[0,1]$ for all $c$, and $k \in \mathbb{R}$. Then:

$$
p l i m \hat{\rho}=\sum_{c=1}^{C} \theta_{c} \rho_{c}+\theta_{0} k .
$$

3. Suppose that $-2<\rho_{c}<0$ for all $c$ (which ensures that the process for each country is stationary), and let $T_{c}$ be the effective number of observations for country $c$. Then, as $\min _{c}\left\{T_{c}\right\} \rightarrow \infty, k, \theta_{c}, \omega_{c}$ and $\hat{\rho}$ all limit to zero (while the limit of $\theta_{0}$ is one).

The proof of this proposition is provided in the Appendix.

Proposition 1 contains several important lessons. First, part 1 of the proposition establishes that the estimate $\hat{\rho}$ does not correspond to a meaningful (local) average, or LATE, of the underlying parameters - the $\rho_{c}$ 's. Some of the $\omega_{c}$ 's in the proposition can be negative, and they need not sum to one. Therefore, one could have cases where $\hat{\rho}$ may be estimated to be zero or positive, even when the underlying (true) $\rho_{c}$ 's are all negative. Part 2 expresses this same result in a different way. By writing the probability limit of $\hat{\rho}$ as the local average of the underlying $\rho_{c}$ 's plus a bias term, it shows that this estimator is inconsistent and biased $]^{3}$ We can also see that this bias is related to the covariance of $\alpha_{c}$ 's with initial conditions (and of course, there is a natural reason for $\alpha_{c}$ to be highly correlated with initial conditions, especially when countries

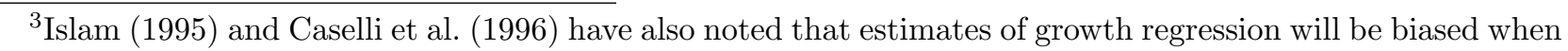
country heterogeneity is not properly controlled for. We are, however, unaware of any other characterization of this bias as in Proposition 1 . 
are close to their steady states). In addition, the bias in question is downward, pushing the coefficient estimate towards zero. $4^{4}$ Third, part 3 shows that as sample size grows, $\hat{\rho}$ will tend to zero - regardless of the true values of the $\rho_{c}$ 's. This sharp result clarifies the intuition for the bias as well: asymptotically, all countries converge to their steady state, and without the $\alpha_{c}$ 's, the only way the model can capture this is by imposing $\hat{\rho}=0$. This bias, which becomes extreme asymptotically, is present for any time length. Finally, because the $\omega_{c}$ 's or the $\theta_{c}$ 's are functions of data moments, the estimate $\hat{\rho}$ (without country fixed effects) will change over time even when the underlying $\rho_{c}$ 's are stable.

We also note that the results in Proposition 11 are relevant beyond Kremer et al.'s paper. These biases and challenges of interpreting the estimates of $\hat{\rho}$ apply to most of the work in the convergence literature.

What do the biases identified in Proposition 1 imply for Kremer et al.'s empirical analysis? We answer this question in the next subsection.

\subsection{Empirical Implications}

To illustrate the issue of the bias identified in Proposition 1, we first estimate (1) - the true DGP under our hypothesis. The estimates of $\rho_{c}$ 's rely on time-series variation in a country's growth rate $5^{5}$ The average number of periods for a country in our sample is 46 .

Figure 1 plots the empirical probability density (Panel A) and the cumulative density (Panel B) of the underlying $\rho_{c}$ 's. We also indicate the estimate $\hat{\rho}$ from equation using a dashed line. A significant bias towards zero in $\hat{\rho}$, as predicted by Proposition 1 , is visible from these results. For example, our estimates of the $\rho_{c}$ 's are negative all the way up to the 88 th percentile, but $\hat{\rho}$ is positive. ${ }^{6}$

Panels C and D of the figure explore the sources of this significant bias in $\hat{\rho}$ by plotting the distributions of the weights $\left(\omega_{c}\right)$ from Proposition 1. Had $\hat{\rho}$ been a meaningful average of the underlying $\rho_{c}$ 's, we would expect all of these weights to be nonnegative. However, in practice, $37 \%$ of them are negative, their sum is also negative $(=-0.024)$, and there are two massive outliers (Costa Rica and Panama)..$^{7}$ This picture confirms that, under the presumption that (1)

\footnotetext{
${ }^{4}$ If $\operatorname{Cov}\left(\varepsilon_{c t}, y_{t-1}\right)=0$, then:

$$
\operatorname{plim} \hat{\rho}=\frac{\operatorname{Cov}\left(\alpha_{c}, y_{c t-1}\right)}{\operatorname{Var}\left(y_{c t-1}\right)}+\sum_{c \in C} \gamma_{c} \rho_{c}
$$

with $\gamma_{c}=\frac{\sum_{t \in T_{c}}\left(y_{c t-1}-\bar{y}_{t-1}\right)^{2}}{\sum_{i \in C} \sum_{t \in T_{i}}\left(y_{i t-1}-\bar{y}_{t-1}\right)^{2}}$ and $\bar{y}_{t-1}=\frac{\sum_{c \in C} \sum_{t \in T_{c}} y_{c, t-1}}{\sum_{c \in C} T_{c}}$.

${ }^{5}$ Like Kremer et al.'s original sample, our data come from the World Development Indicators database. As noted in footnote 1. the final version of their paper uses data from the Penn World Tables. The two datasets give very similar results. We keep the original data in our analysis in the text and report analogous results with the Penn World Tables in the Appendix.

${ }^{6} \mathrm{As}$ in all of the other empirical models we report in this paper, the country fixed effects, the $\alpha_{c}$ 's, are highly significant when we estimate these country-specific convergence parameters. In Panel A, for example, the F-statistic for their joint significance is 276,182 .

${ }^{7}$ With the Penn World Tables data, Costa Rica and Panama are no longer outliers, but now Belarus is a
} 
is the true model/DGP, estimates of $\hat{\rho}$ do not correspond to economically meaningful objects: even when the vast majority of countries are converging, $\hat{\rho}$ may create the impression that there is no convergence.

We now turn to part 2 of Proposition 1 and explore where the bias of $\hat{\rho}$ comes in the sample studied by the authors. The next equation summarizes the results by presenting the empirical counterparts of the decomposition given in part 2 of Proposition 1 :

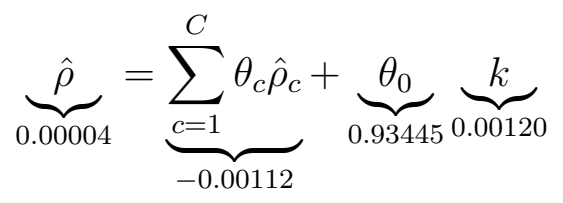

The advantage of this equation is that it provides a decomposition of $\hat{\rho}$ into an average of the underlying parameters (the first term) and the bias (the second term). Recall, in particular, that $\theta_{c} \in[0,1]$ and $\sum_{c=0}^{C} \theta_{c}=1$. In our sample, we find that $\sum_{c=1}^{C} \theta_{c} \hat{\rho}_{c}=-0.00112$, suggesting that at least on average, most country-specific coefficients of convergence tend to be negative (in line with $88 \%$ of them being negative as we saw above). However, the coefficient of unconditional convergence $\hat{\rho}$ is positive $(=0.00004)$, because the second term is very large - comprised of a huge weight $\theta_{0}=0.93445$ and a positive bias $k=0.00120$.

We now turn to the question of what accounts for over-time changes in $\hat{\rho}$. In Figure 2, we report the same decomposition from part 2 of Proposition 1, but now separately by decade. Consistent with Kremer et al., we find that estimates of $\hat{\rho}$ from (2) are decreasing over time, as shown by the green bars. Strikingly, however, this pattern is entirely driven by changes in the bias term (shown by the blue bar). The underlying distribution of the $\rho_{c}$ 's (shown by the orange bar) has remained quite stable over time. Therefore, all of the dynamics in the authors' estimate of $\hat{\rho}$ seem to come from the bias term rather than from a faster rate of economic convergence.

We also explored the determinants of convergence patterns for the key covariates used by Kremer et al. 3 Table 1 reports the coefficient estimate $\hat{\rho}$ from equation (2) as well as key statistics about the distribution of estimates of the underlying $\rho_{c}$ 's estimated from (1). In all cases, estimates of $\hat{\rho}$ are significantly above the mean or the median of the distribution of the estimates of the $\rho_{c}$ 's (and except for Polity 2, they are also above the 75th percentile). These results again underscore that estimates that ignore country heterogeneity are going to be significantly biased towards zero.

massive outlier. The rest of the results are very similar. See Figure A-1 in the Appendix.

${ }^{8}$ These are (source in parenthesis): Polity 2 (Polity IV Project), Rule of law (Worldwide Governance Indicators), Property rights (Heritage Freedom), Government expenditure (World Development Indicators) and Years of schooling (Barro-Lee). 


\section{Estimating the Effects of Institutions and Policies}

In the second part of their paper, Kremer et al. add the covariates mentioned in the previous section to the right hand side of (2) and assume that they have the same impact across countries, while still not including country fixed effects. As they again consider 10-year changes on the left-hand side, their model is now

$$
\Delta_{10} y_{c t}=\beta_{t} x_{c t}+\rho y_{c t}+\delta_{t}+\varepsilon_{c t},
$$

where $\Delta_{10} y_{c t}=y_{c t+10}-y_{c t}$.

Estimating this equation on the same sample, they conclude that conditional convergence patterns have been stable over the sample and the change in unconditional convergence implied by their estimates of $\hat{\rho}$ are due to the correlation between growth and institutions (or policies or other country characteristics) having become weaker over time. They also report that their

estimates of $\beta$ from this equation, $\hat{\beta}$, are getting smaller over time, which they interpret as the relationship between these factors and economic growth becoming weaker.

Although in the revision of their paper the authors recognize that these estimates are not causal and should be interpreted with caution, the same issues highlighted in the previous section are still relevant and, as we will see, are responsible for the majority of their results. In the rest of this section, we develop this point by focusing on one dimension of institutions that has been explored in detail in the literature and found to be robustly related to growth: democracy (see Acemoglu et al. 2019). Before doing this, however, in the next subsection, we highlight that even ignoring these problems, the data do not unambiguously point out to a decline in $\beta_{t}$.

\subsection{The Importance of Horizons}

As noted above, Kremer et al. use the 10-year change on the left-hand side of (3) and then compare the estimates for 1985 and 2005. In Panel A of Figure 3, we reestimate their model, still with 10-year changes, but separately by year. We focus on their main measure of democracy, Polity 2 score. Indeed the coefficient estimates from this model are greater for 1985 and 2005, but the pattern we see is not one of decline, but a cyclical one. This suggests that whatever is driving the authors' results may be more complex than a simple secular flattening.

Panel B of the same figure reestimate the authors' model, but using annual changes on the left-hand side. Now, although the estimates do change over time, there is no evidence of a decline. We conjecture, instead, that the patterns shown in Panel A are driven by the interaction of the changing bias terms, highlighted in the previous section, and the 10-year horizon on the left-hand side, which cumulates these changes. We explore whether the effects of institutions are indeed diminishing over time more systematically in the next subsection. 


\subsection{Have the Causal Effects of Institutions Declined Over Time?}

We now study this question, focusing on the effects of democracy. We follow Acemoglu et al. (2019) in focusing on a dichotomous measure of democracy (to minimize measurement error) and extend (1) to include several lags of GDP per capita on the right-hand side, and as in their paper, simplify the model by assuming constant effects of democracy and the lags, but, in line with the focus here, we allow the effect of democracy to be time-varying. This gives:

$$
\ln y_{c t}=\beta_{t} D_{c t}+\sum_{j=1}^{p} \rho_{j} \ln y_{c t-j}+\alpha_{c}+\delta_{t}+\varepsilon_{c t},
$$

where $D_{c t}$ denotes the dichotomous measure of democracy (dictatorship vs. democracy). All the other variables are as in (1), and crucially, as in that equation, we have country fixed effects, represented by the $\alpha_{c}$ 's.

First, suppose that $\beta_{t}=\beta$. Then, under sequential exogeneity, the within-estimator of this equation recovers an interpretable estimate of an economically-meaningful object, $\beta !^{9}$ We also report alternative estimators (including IV estimate using as instrument waves of democratization), which are consistent under related but slightly different assumptions 10

We first replicate the results of Acemoglu et al. (2019), estimating a time-invariant $\beta$ in equation (4). The results are reported in Panel A of Table 2. All regressions include four lags of log GDP. As in Acemoglu et al. (2019), and common with all of the other results we will report, the democracy variable is estimated to have a precise and significant positive impact on GDP per capita, with a coefficient of 0.787 (standard error $=0.226$ ) in the OLS specification in column 1. This estimate implies that a permanent transition to democracy leads to an approximately $20 \%$ increase in GDP per capita after about 25 years 11 As in the original results in Acemoglu et al. (2019), the other columns, including the IV procedure exploiting regional waves of democratization, show similar estimates.

Have these effects changed over time? To answer this question in the simplest possible way, we parameterize $\beta_{t}$ in equation (4) as a linear function of time, which is equivalent to including an interaction between the democracy score and time, $D_{c} t$, as an additional regressor. Results from this exercise are reported in Panel B. In all cases, this interaction has a positive coefficient, and shows no evidence of a decline over time. For example, with OLS in column 1 the coefficient estimate is 0.383 (standard error $=0.246$ ), while with IV in column 4, it is 0.716 (standard error $=0.368$ ), which is significant at $10 \%$. We therefore conclude that, once one focuses on a model that allows for unobserved country heterogeneity which proves to be important in all of these

\footnotetext{
${ }^{9}$ Sequential exogeneity requires that $\mathbb{E}\left[\varepsilon_{c t} \mid y_{c t-1}, \ldots, y_{c t_{0}}, D_{c t}, \ldots, D_{c t_{0}}, \alpha_{c}, \delta_{t}\right]=0$ for all $y_{c t-1}, \ldots, y_{c t_{0}}, D_{c t}, \ldots, D_{c t_{0}}, \alpha_{c}$ and $\delta_{t}$ and for all $c$ and $t \geq t_{0}$.

${ }^{10}$ See Acemoglu et al. (2019) for a discussion of these assumptions.

${ }^{11}$ The F-statistic for the significance of country fixed effects in the specification is 1417, again indicating that these fixed effects are highly significant.
} 
specifications, there is no evidence of the causal effect of democracy to have declined over time. ${ }^{12}$

\subsection{Squaring the Circle}

The model in equation (4) also gives us an opportunity to unify the two parts of our analysis so far-relating to changes in convergence patterns and changes in the effects of institutions. We now estimate this equation separately by decade and report the implied convergence estimates $\hat{\rho}$ (assumed to be the same across countries for this exercise). We focus on four specifications, which are all reported in Figure 4. The first is Kremer et al.'s specification of unconditional convergence, which does not include country fixed effects (shown by the black bars the left axis). The second is a specification that adds country fixed effects to this baseline (shown by the orange bars on the right axis). The third and fourth add our dichotomous measure of democracy as a control, with constant and heterogeneous effects across countries, respectively (shown by the blue and green bars on the right axis).

The black bars confirm the pattern emphasized by the authors: in the 1970s, 80s and 90s, the estimate of $\hat{\rho}$ is positive and then declines to be negative in the 2000s and 2010s. However, the remaining bars show that this pattern is driven entirely by the absence of country fixed effects. As soon as these are included, the orange bars are uniformly negative and do not show a clear trend either way. The same pattern is visible with the blue and green bars. Another noteworthy feature, highlighting the downward bias in the converges coefficient without fixed effects identified in Proposition 1, is that the coefficient estimates shown by the black bars are about $1 / 50$ th of the others, and hence the need for having two vertical axes to be able to depict these estimates 13

We conclude once again that the time-trends in the estimates of $\hat{\rho}$ in equation (2) do not appear to be related to a change in the causal relationship between economic growth and its country-level determinants.

\section{Authors' Response to our Comments}

In response to our conference discussion, the published version of Kremer et al. includes various adjustments (in addition to the change of dataset we noted above). We welcome several of these adjustments. They estimate an augmented version of their model in the Appendix, which allows for fixed effects and confirm our results that there is no major change in convergence

\footnotetext{
${ }^{12}$ All of the results reported so far are very similar using data from the Penn World Tables, see Appendix A.2

${ }^{15}$ The only difference with the Penn World Tables comes in this analysis, where the analogues of the black bars in this figure show even less precision and are not uniformly positive before the 2000s. However, the other aspects of this figure and the overall conclusion from this alternative dataset are identical. See Figure A-4 in the Appendix.
} 
patterns in this case. However, the authors argue against including country fixed effects. They allow these fixed effects to vary across decades and then plot these decadal fixed effect estimates against each other. They state that there is little correlation between decadal fixed effects and conclude that this lack of correlation "call[s] into question the benefit of a model including fixed effects."

We reproduce this exercise in Figure 5. Our results are very different from theirs. We find that the fixed effect estimates are highly persistent and the correlation between fixed effects in different decades is always above $95 \%$. This is true regardless of whether we compare fixed effects for a decade starting with year $t$ vs $t+10$ or $t+20$, and regardless of the exact specification of the dependent variable (Panel A is for models with first differences of GDP per capita on the left-hand side, while Panel B is for models with 10-year changes) ${ }^{14}$ These patterns, combined with the very high F-statistics for the significance of the country fixed effects in all of our models, indicate that these fixed effects belong in the models and their omission is responsible for estimates that are difficult to interpret in terms of underlying economic effects.

\section{Conclusion}

Kremer et al. is a timely paper revisiting the evolution of convergence cross-country patterns over the last six decades. The authors provide evidence that the lack of convergence that applied early in the sample has now been replaced by modest convergence. They also argue this relationship is driven by convergence in various determinants of economic growth across countries and a flattening of the relationship between these determinants and growth. Although the patterns documented by the authors are intriguing, our reanalysis finds that these results are driven by the lack of country fixed effects controlling for unobserved determinants of GDP per capita across countries. We establish theoretically that failure to include for such potential determinants will create a bias in convergence coefficients towards zero and, equally importantly, the resulting estimates may not have straightforward economic interpretations (for example, they will not correspond to any type of local average of the effects at the country level). The root cause of this bias is simple: when there are permanent differences across countries and each country is close to its steady state, a model that does not include fixed effects can only fit the data by having a convergence coefficient very very close to zero. This point is of more general relevance, since it applies not just to Kremer et al.'s study, but also to the majority of the convergence literature.

Empirically, we show that estimated convergence coefficients (from models that do not include fixed effects) are indeed biased towards zero. Moreover, this bias is time-varying, even though the underlying country-level parameters appear to be constant and stable.

\footnotetext{
${ }^{14}$ These exercises should still be interpreted with caution. As is well known, estimates of fixed effects are inconsistent for finite $T$ (Wooldridge, 2010).
} 
The authors' finding that the relationship between economic growth and its country-level determinants (such as institutions) is flattening is as notable. If true, it might suggest that improving institutions and policies may have become less important for explaining and spearheading growth. It might also have important policy implications. However, our reanalysis finds no evidence of a flattening in the relationship between institutional variables and economic growth. Focusing on democracy, we show that this variable's impact continues to be precisely estimated and, if anything, a little larger than the beginning of the sample.

\section{References}

Acemoglu, D., Naidu, S., Restrepo, P., \& Robinson, J. A. (2019). Democracy does cause growth. Journal of Political Economy, 127(1), 47-100.

Arellano, M., \& Bond, S. (1991). Some tests of specification for panel data: Monte Carlo evidence and an application to employment equations. The Review of Economic Studies, $58(2), 277-297$.

Barro, R. J. (1991). Economic growth in a cross section of countries. The Quarterly Journal of Economics, 106(2), 407-443.

Barro, R. J., \& Sala-i Martin, X. (1992). Convergence. Journal of Political Economy, 100(2), $223-251$.

Caselli, F., Esquivel, G., \& Lefort, F. (1996). Reopening the convergence debate: a new look at cross-country growth empirics. Journal of Economic Growth, 1(3), 363-389.

Hahn, J., Hausman, J. A., \& Kuersteiner, G. M. (2001). Bias corrected instrumental variables estimation for dynamic panel models with fixed effects. Available at SSRN 276592.

Islam, N. (1995). Growth empirics: a panel data approach. The Quarterly Journal of Economics, $110(4), 1127-1170$.

Knight, M., Loayza, N., \& Villanueva, D. (1993). Testing the neoclassical theory of economic growth: a panel data approach. IMF Staff Papers, 40(3), 512-541.

Kremer, M., Willis, J., \& You, Y. (2021). Converging to convergence. NBER Macro Annual 2021.

Loayza, N. (1994). A test of the international convergence hypothesis using panel data. World Bank Publications, Policy Research Working Paper, 1333.

Wooldridge, J. M. (2010). Econometric analysis of cross section and panel data. MIT press, Cambridge. 


\section{Figures and Tables}

\section{Figure 1: Empirical distribution of the underlying $\rho_{c}$ 's and $\omega_{c}$ 's}

Panel A: Probability density of the $\rho_{c}$ 's

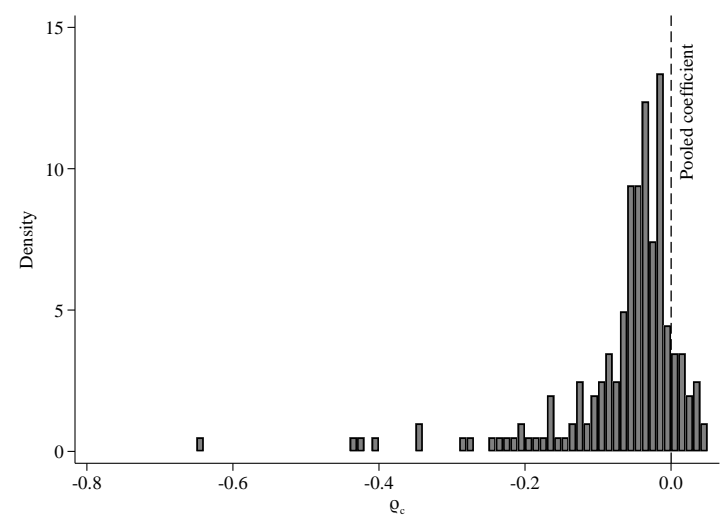

Panel C: Probability density of the $\omega_{c}$ 's

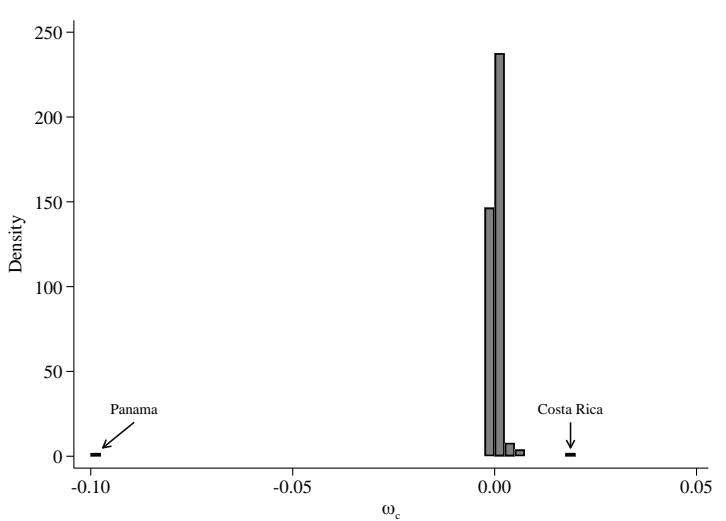

Panel B: Cumulative density of the $\rho_{c}$ 's

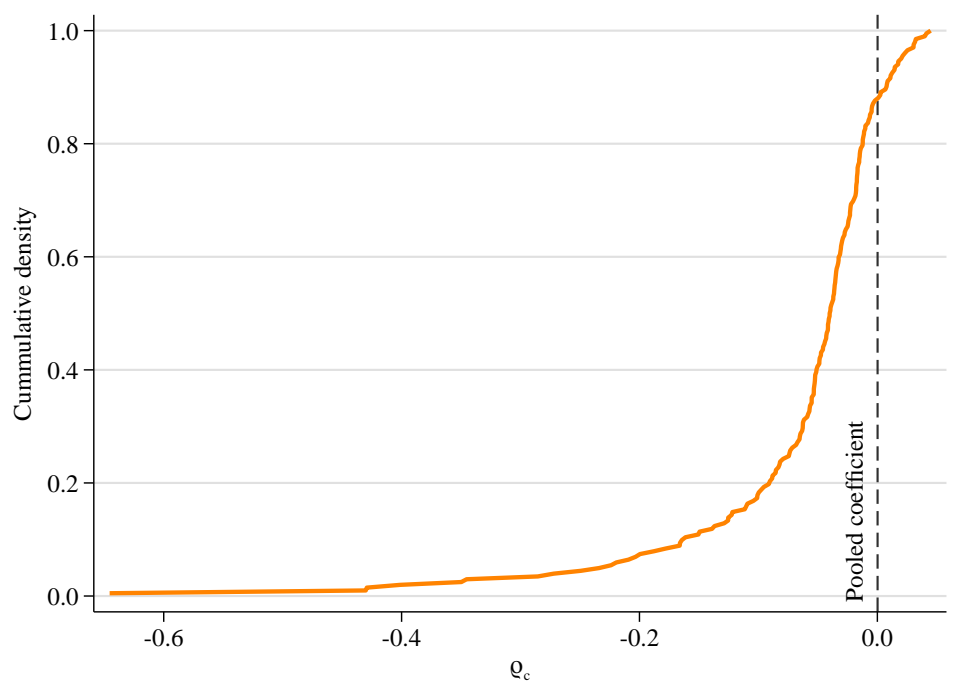

Panel D: Cumulative density of the $\omega_{c}$ 's

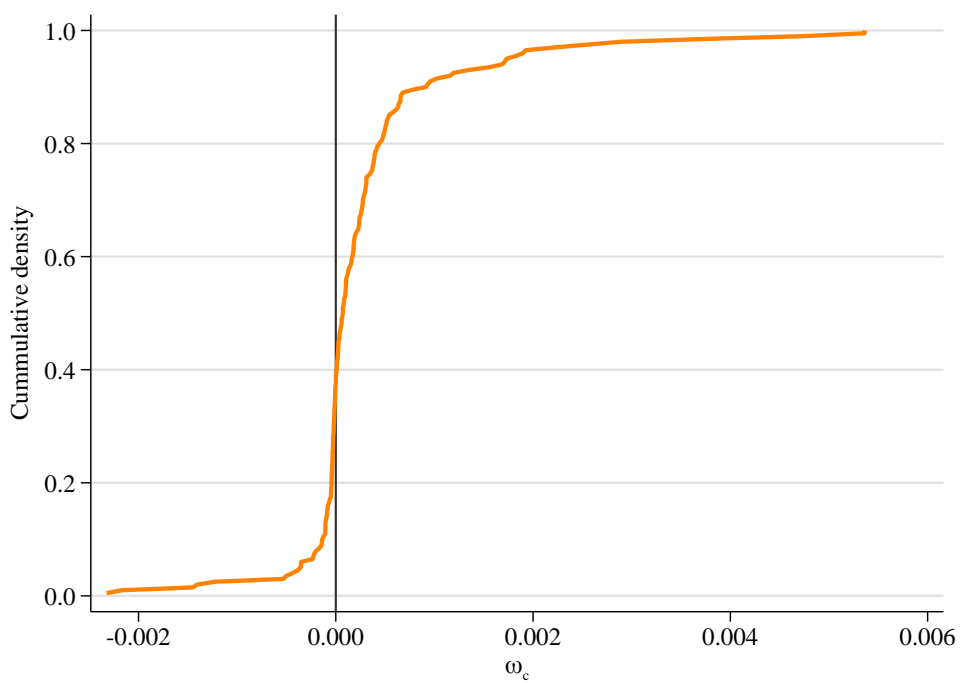

Notes: The figure presents estimates of the distribution of the country-specific coefficients of convergence (the $\rho_{c}$ 's), and the weights (the $\omega_{c}$ 's, defined in Proposition 1]. The empirical probability density and the cumulative density are reported on the left and the right-hand side panels respectively. In Panel A, the dashed line indicates the estimate $\hat{\rho}$ from equation 2 (when no country heterogeneity is allowed). 
Figure 2: Decomposition of $\hat{\rho}$ across decades

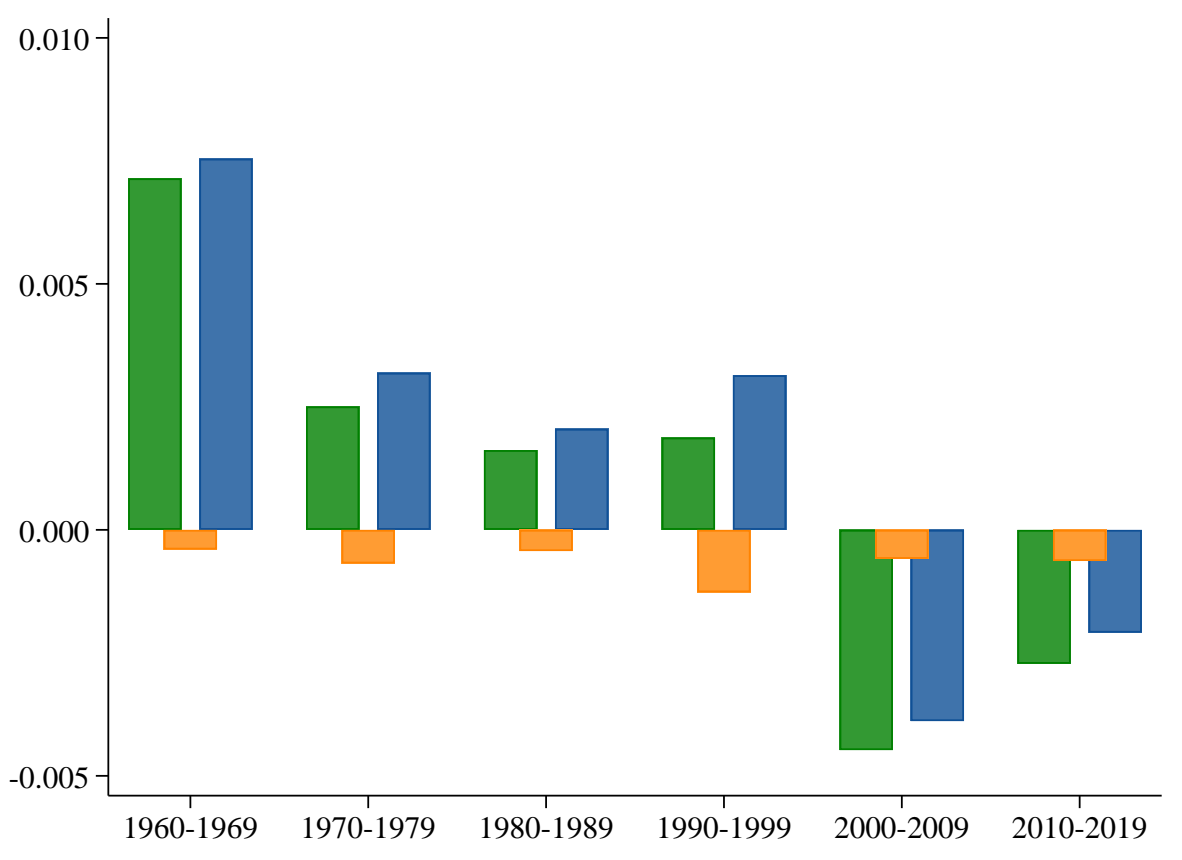

Notes: The figure reports the estimate $\hat{\rho}$ from equation 22. (when no country heterogeneity is allowed) by decade shown by the blue bar. This estimate is decomposed in two terms (see Proposition 1): the underlying distribution of the $\rho_{c}$ 's shown by the orange bar and the bias shown by the green bars. 
Figure 3: Estimates of the relationship of Polity 2 and economic growth over time

Panel A: Dependent variable is 10-year changes

$\Delta_{10} y_{c t}=\beta_{t} x_{c t}+\rho_{t} y_{c t}+\delta_{t}+\varepsilon_{c t}$

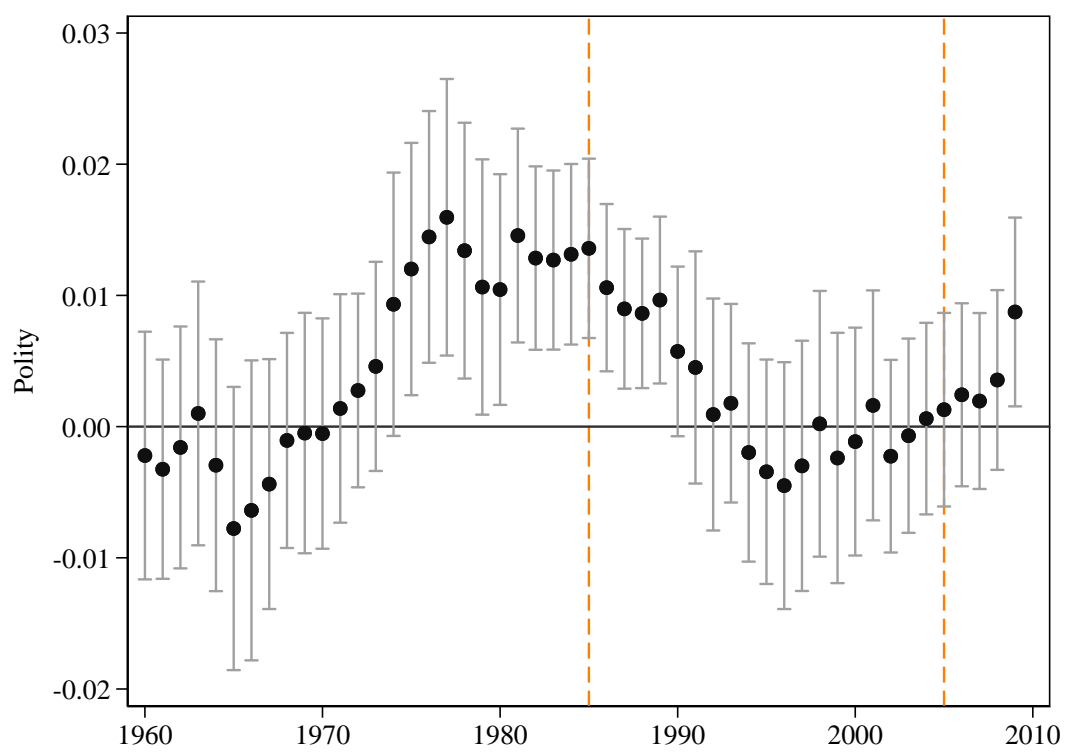

Panel B: Dependent variable is first difference

$\Delta y_{c t}=\beta_{t} x_{c t}+\rho_{t} y_{c t}+\delta_{t}+\varepsilon_{c t}$

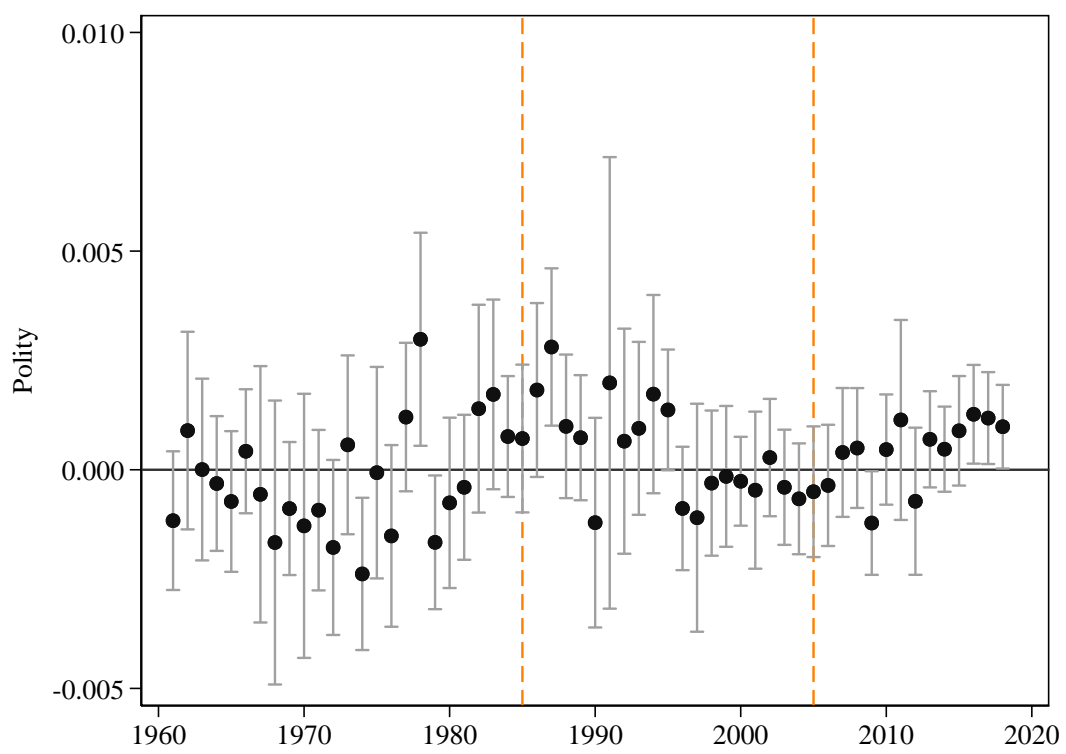

Notes: The figure reports estimates of the relationship between the Polity 2 score and economic growth and $95 \%$ confidence intervals over time, Namely, we plot the coefficient $\beta_{t}$ in the equation indicated in the panel labels. Panel A uses as dependent variable the 10-year change in log of GDP per capita, while Panel B uses annual changes (first differences). Standard errors are clustered at the country level. 


\section{Figure 4: Estimates of $\rho$ across different specification and decades}

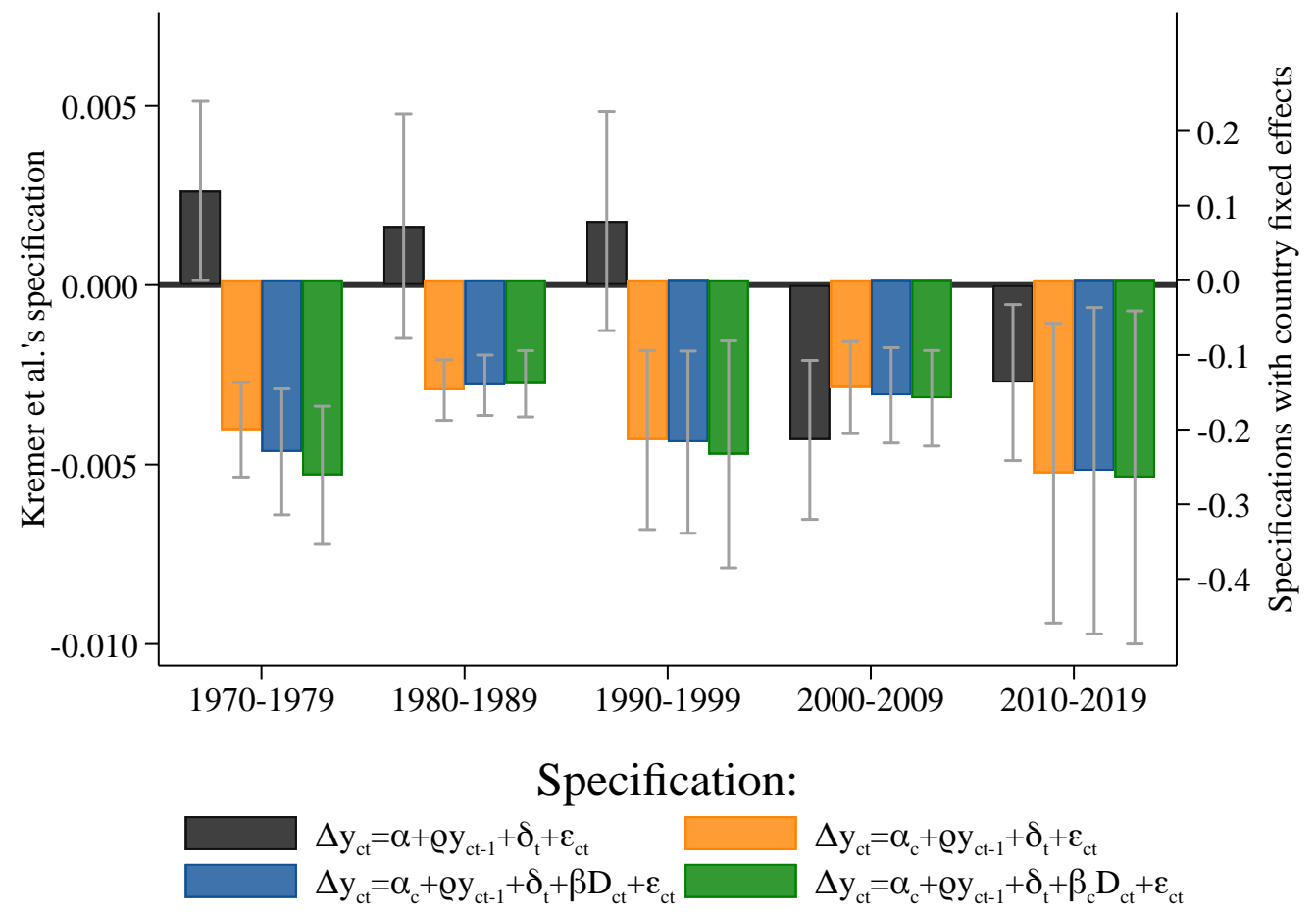

Notes: The figure report estimates of the coefficient of convergence $(\rho)$ as well as $95 \%$ confidence intervals across four different specifications. The first is Kremer et al. s specification of unconditional convergence, which does not include country fixed effects (shown by the black bars). The second is a specification that adds country fixed effects to this baseline (shown by the orange bars). The third adds our dichotomous measure of democracy as a control, focusing on the OLS specification (shown by blue bars). Our final specification allows the effects of democracy to be varying across countries green bars). Standard errors are clustered at the country level. 


\section{Figure 5: Correlation of country fixed effects over decades}
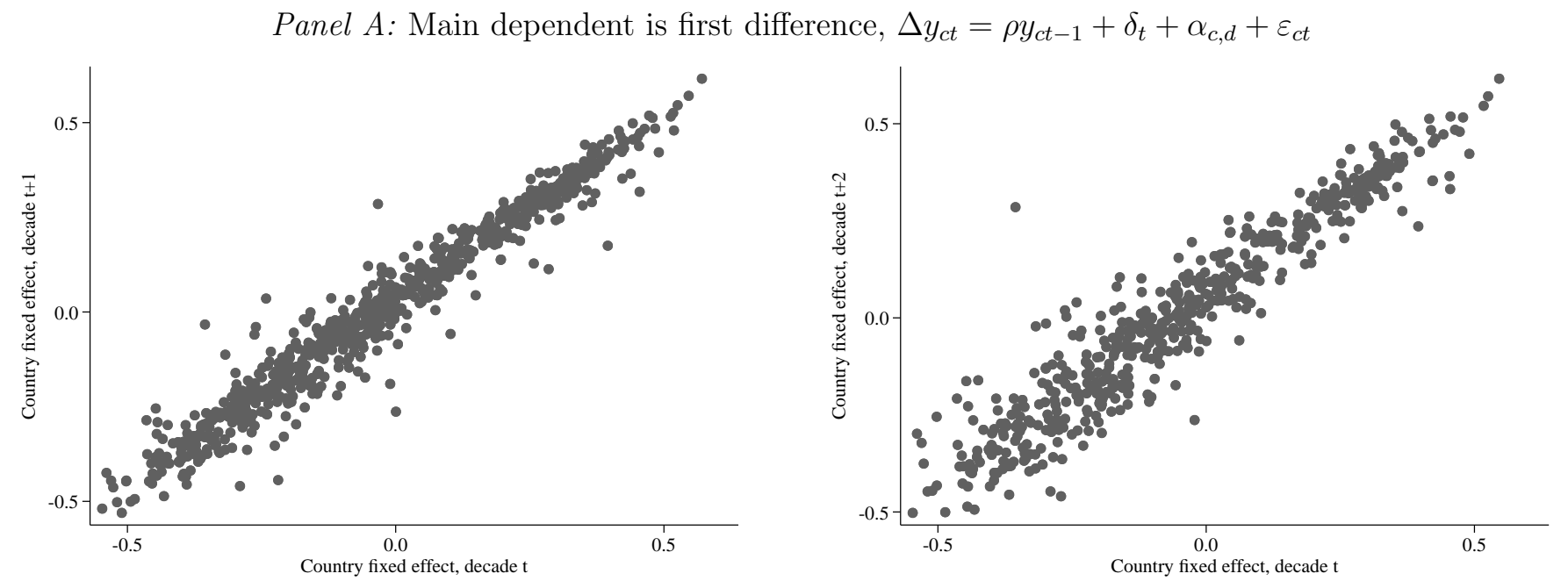

Panel B: Main dependent variable is 10-year changes, $y_{c, t+10}-y_{c, t}=\rho y_{c t}+\delta_{t}+\alpha_{c, d}+\varepsilon_{c t}$
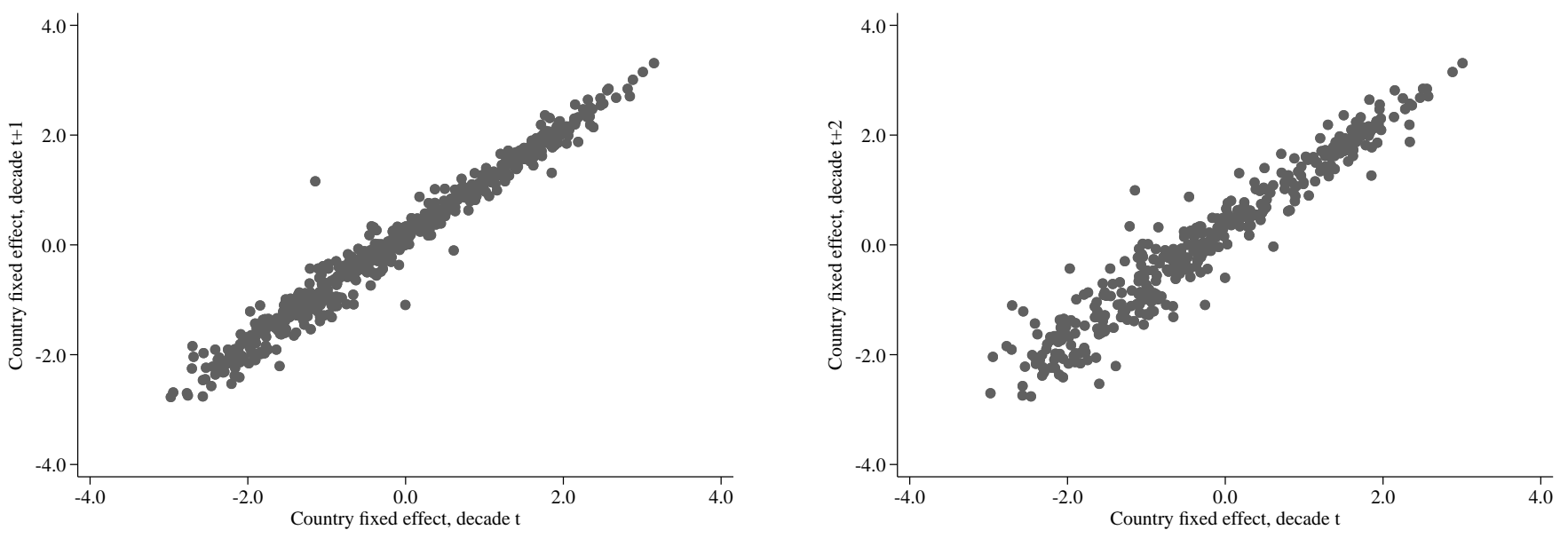

Notes: We estimate the regression specified in the panel label which allows the country fixed effects to vary across decades and then plot these decadal fixed effect estimates against each other. Panels A and B use as dependent variable the first difference (the 10-year change) in log of GDP per capita, while Panels C and D use 10-year changes. The panels to the left (right) compare shows the correlation between the estimated country fixed effect for a decade starting at year $\mathrm{t}$ with a decade starting at year $\mathrm{t}+10(\mathrm{t}+20)$. 
Table 1: Convergence patterns for the key covariates

\begin{tabular}{lccccc}
\hline \hline & $(1)$ & $(2)$ & $(3)$ & $(4)$ & $(5)$ \\
& Pooled & \multicolumn{4}{c}{ Country-level coefficients } \\
\cline { 3 - 5 } & coefficient & Mean & 25th quantile & 50th quantile & 75th quantile \\
\hline \multirow{2}{*}{ Log GDP per capita } & 0.000 & -0.063 & -0.074 & -0.040 & -0.017 \\
Polity 2 & -0.029 & -0.107 & -0.165 & -0.087 & 0.000 \\
Rule of law & -0.008 & -0.346 & -0.487 & -0.295 & -0.169 \\
Property rights & -0.032 & -0.239 & -0.360 & -0.228 & -0.133 \\
Government expenditure & -0.055 & -0.215 & -0.313 & -0.168 & -0.069 \\
Credit & 0.004 & -0.221 & -0.404 & -0.114 & -0.013 \\
Years of schooling & -0.020 & -0.506 & -0.653 & -0.440 & -0.321 \\
\hline \hline
\end{tabular}

Notes: The table reports estimates of coefficient of unconditional convergence ( $\rho$, see equation $\sqrt{2}$ ) $)$ in column 1. The remaining columns report moments (including the mean and the 25th, 50th and 75th quantiles) of the distribution of the underlying country-specific estimates of convergence $\left(\rho_{c}\right.$ 's, see equation (10). We show results for the key variables used by Kremer et al. including (source in parenthesis): Polity 2 (Polity IV Project), Rule of law (Worldwide Governance Indicators), Property rights (Heritage Freedom), Government expenditure (World Development Indicators) and Years of schooling (Barro-Lee). 


\section{Table 2: Estimates of the Effect of democracy on (log) GDP per capita}

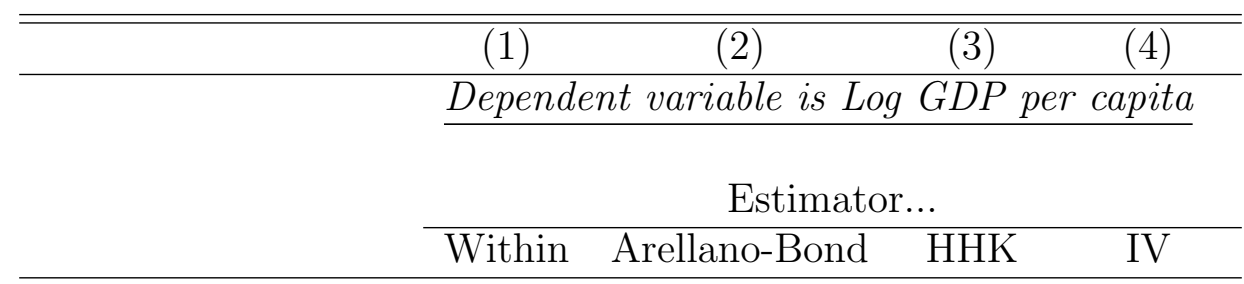

\section{A. Effect of Democracy on Log GDP}

\begin{tabular}{lcccc} 
Democracy & 0.787 & 0.875 & 1.165 & 0.966 \\
& $(0.226)$ & $(0.374)$ & $(0.370)$ & $(0.562)$ \\
Observations & 6,336 & 6,161 & 6336 & 6,312 \\
\hline
\end{tabular}

B. Effect of Democracy on Log GDP and its change over time

\begin{tabular}{lcccc}
\hline \multirow{2}{*}{ Democracy } & & & & \\
& 0.678 & 0.872 & 0.886 & 1.346 \\
Democracy $\times$ Trend & $0.214)$ & $(0.374)$ & $(0.394)$ & $(0.616)$ \\
& 0.383 & 0.270 & 0.442 & 0.716 \\
Observations & $(0.246)$ & $(0.362)$ & $(0.357)$ & $(0.368)$ \\
\hline \hline
\end{tabular}

Notes: This table presents estimates of the effect of democracy on log GDP per capita following Acemoglu et al. (2019). Democracy is measured as a dichotomous variable to minimize measurement error. Panel A replicates the results in Acemoglu et al. (2019), and Panel B extends the regression by allowing an interaction between the measure of democracy and a linear function of time. Column 1, 2, 3 and 4 present results from the within estimator, the Arellano and Bond/s (1991) GMM estimator, the HHK (Hahn, Hausman, \& Kuersteiner, 2001) estimator, and an IV (exploiting regional waves of democratization) respectively. All regressions include four lags of log GDP per capita. Standard errors are clustered at the country level. 


\section{A Appendix for Acemoglu and Molina (Not For Publi- cation)}

\section{A.1 Proof of Proposition 1}

Let $c=\{1,2, \ldots, C\}$ designate a country and $C$ be the total number of countries. Suppose that the main outcome of interest, $y_{c t}$, follows the $\operatorname{AR}(1)$ process

$$
\Delta y_{c t}=\alpha_{c}+\rho_{c} y_{c t-1}+\varepsilon_{c t}
$$

so that the joint data generating process for all countries follows equation (1). Let $T_{c}=\left\{t \mid y_{c, t} \in\right.$ $\left.\mathbb{R} \wedge y_{c, t-1} \in \mathbb{R}\right\}$. Let us first consider the OLS estimate of country-specific persistent parameter $\hat{\rho}_{c}$ for country $c$ (with country-specific intercepts). This can be written as:

$$
\hat{\rho}_{c}=\frac{\sum_{t \in T_{c}}\left(\Delta y_{c}-\overline{\Delta y}_{c}\right)\left(y_{c, t-1}-\bar{y}_{c,-}\right)}{\sum_{t \in T_{c}}\left(y_{c, t-1}-\bar{y}_{c,-}\right)^{2}},
$$

where $\overline{\Delta y}_{c}=\frac{\sum_{t \in T_{c}} \Delta y_{c, t}}{T_{c}}$ and $\bar{y}_{c,-}=\frac{\sum_{t \in T_{c}} y_{c, t-1}}{T_{c}}$. Because the DGP is given by (1), we have $\operatorname{plim} \hat{\rho}_{c}=\rho_{c}$.

Now consider the OLS estimate of $\rho, \hat{\rho}$, in the cross-country equation of unconditional convergence without country-specific intercepts (country fixed effects) as in equation (2):

$$
\hat{\rho}=\frac{\sum_{c \in C} \sum_{t \in T_{c}}\left(\Delta y_{c, t}-\overline{\Delta y}\right)\left(y_{c, t-1}-\bar{y}_{-}\right)}{\sum_{c \in C} \sum_{t \in T_{c}}\left(y_{c, t-1}-\bar{y}_{-}\right)^{2}},
$$

where $\overline{\Delta y}=\frac{\sum_{c \in C} \sum_{t \in T_{c}} \Delta y_{c, t}}{\sum_{c \in C} T_{c}}$ and $\bar{y}_{-}=\frac{\sum_{c \in C} \sum_{t \in T_{c}} y_{c, t-1}}{\sum_{c \in C} T_{c}}$.

We first prove part 2 of the proposition, thus establishing that

$$
\hat{\rho}=\sum_{c=1}^{C} \theta_{c} \hat{\rho}_{c}+\theta_{0} k
$$

To do so, we expand equation (6), and write the right-hand side, in terms of the $\hat{\rho}_{c}$ 's from equation (5). The numerator of this expression can be written as

$$
\begin{aligned}
\sum_{c \in C} \sum_{t \in T_{c}}\left(\Delta y_{c, t}-\overline{\Delta y}\right)\left(y_{c, t-1}-\bar{y}_{-}\right) & =\sum_{c \in C} \sum_{t \in T_{c}} \Delta y_{c, t}\left(y_{c, t-1}-\bar{y}_{-}\right) \\
& =\sum_{c \in C} \sum_{t \in T_{c}} \Delta y_{c, t}\left(y_{c, t-1}-\bar{y}_{c,-}\right)+\sum_{c \in C} \sum_{t \in T_{c}} \overline{\Delta y}_{c}\left(\bar{y}_{c,-}-\bar{y}_{-}\right) \\
& =\sum_{c \in C} \sum_{t \in T_{c}} \Delta y_{c, t}\left(y_{c, t-1}-\bar{y}_{c,-}\right)+\sum_{c \in C} T_{c} \overline{\Delta y}_{c}\left(\bar{y}_{c,-}-\bar{y}_{-}\right)
\end{aligned}
$$

where the first equality uses the fact that $\sum_{t \in T_{c}}\left(y_{c, t-1}-\bar{y}_{-}\right)=0$, while the second equality 
rearranges terms. The final equality follows from the fact that $\overline{\Delta y}_{c}, \bar{y}_{c,-}$, and $\bar{y}_{-}$do not vary with $t$. Analogously, we can rewrite the denominator as

$$
\sum_{c \in C} \sum_{t \in T_{c}}\left(y_{c, t-1}-\bar{y}_{-}\right)^{2}=\sum_{c \in C} \sum_{t \in T_{c}}\left(y_{c, t-1}-\bar{y}_{c,-}\right)^{2}+\sum_{c \in C} T_{c}\left(\bar{y}_{c, t-1}-\bar{y}_{-}\right)^{2} .
$$

Now, letting $\sigma_{c}=\sum_{t \in T_{c}}\left(y_{c t-1}-\bar{y}_{c-}\right)^{2}$, equation (6) can be written as

$$
\begin{aligned}
\hat{\rho} & =\frac{\sum_{c \in C} \sigma_{c} \hat{\rho}_{c}+\sum_{c \in C} T_{c} \overline{\Delta y}_{c}\left(\bar{y}_{c,-}-\bar{y}_{-}\right)}{\sum_{c \in T_{c}} \sigma_{c}+\sum_{c \in C} T_{c}\left(\bar{y}_{c, t-1}-\bar{y}_{t-1}\right)^{2}} \\
& =\sum_{c=1}^{C} \theta_{c} \hat{\rho}_{c}+\theta_{0} k
\end{aligned}
$$

whereon

$$
\begin{aligned}
\theta_{c} & =\frac{\sigma_{c}}{\sum_{c \in C} \sigma_{c}+\sum_{c \in C} T_{c}\left(\bar{y}_{c,-}-\bar{y}_{-}\right)^{2}} \forall c=\{1, \ldots, C\} \\
\theta_{0} & =1-\sum_{c=1}^{C} \theta_{c} \\
k & =\frac{\sum_{c \in C} T_{c} \overline{\Delta y}_{c}\left(\bar{y}_{c,-}-\bar{y}_{-}\right)}{\sum_{c \in C} T_{c}\left(\bar{y}_{c,-}-\bar{y}_{-}\right)^{2}} .
\end{aligned}
$$

Since $\operatorname{plim} \hat{\rho}_{c}=\rho_{c}$, we can substitute $\rho_{c}$ for $\hat{\rho}_{c}$ in the limit, which gives us the desired result. Note also that $\sigma_{c} \geq 0$ and thus $\theta_{c} \in[0,1]$.

To prove part 1 of the proposition, assume $\hat{\rho}_{c} \neq 0 \forall c$, and then substitute

$$
\theta_{c}=\omega_{c}-\frac{\theta_{0} k}{C \hat{\rho}_{c}}
$$

in equation (7) and simplify terms. It is straightforward to see that $\omega_{c}$ need not be between 0 and 1 , and $\sum_{c=1}^{C} \omega_{c}$ is not in general equal to one. Our empirical results in Section 3.2 provide one instance in which $\omega_{c}$ is not between 0 and 1 for a significant fraction of countries in the sample, and their sum is very different from 1.

Finally, we establish part 3 by proving that, for any $c, \lim _{T_{c} \rightarrow \infty} \sigma_{c} \in \mathbb{R}$. Let us drop the country subscript $c$, since we will work with the equation for a single country: $\Delta y_{t-1}=$ $\alpha+\rho y_{t-2}+\varepsilon_{t-1}$, or

$$
y_{t-1}=\alpha+\gamma y_{t-2}+\varepsilon_{t-1},
$$

where $\gamma=\rho+1$. Substituting successively, the solution to this equation takes the form

$$
y_{t-1}=a+b \gamma^{t-1}+\varepsilon_{1} \gamma^{t-2}+\varepsilon_{2} \gamma^{t-3}+\ldots+\varepsilon_{t-1},
$$


where $b=y_{0}-a$ and $a=\frac{\alpha}{1-\gamma}$ ( $a$ represents the steady state and $b$ is the distance of the initial value to the steady state). Then

$$
\begin{aligned}
\sigma & =\sum_{t \in T}\left(y_{t-1}-\bar{y}_{t-1}\right)^{2} \\
\sigma & =\sum_{t \in T} y_{t-1}^{2}-\frac{1}{T}\left(\sum_{t=1}^{T} y_{t-1}\right)^{2} \\
\sigma & =a^{2} T+O_{p}(1)-\left[a^{2} T+O_{p}(1)\right] \\
\sigma & =O_{p}(1)
\end{aligned}
$$

where the second equality follows by rearranging terms. The third equality follows from substituting for equation (10) and using the hypothesis that $-2<\rho_{c}<0$, or equivalently $|\gamma|<1$, which implies that $y_{t-1}$ converges, and thus both $\sum_{t \in T} y_{t-1}^{2}$ and $\frac{1}{T}\left(\sum_{t=1}^{T} y_{t-1}\right)^{2}$ are $O_{p}(T)$. The fourth equality simply notes that their difference is $O_{p}(1)$, which also implies that the limit of $\sigma$ is finite.

The final step is to characterize $p \lim \theta_{c}$. The result above implies that if $\underline{T}=\min _{c}\left\{T_{c}\right\} \rightarrow \infty$, then $\sigma_{c}$ is finite for any $c$. Additionally, both $\bar{y}_{c,-}$ and $\bar{y}_{-}$converge to limit values (since each country converges to its steady state, again my hypothesis). However, because of the $\alpha_{c}$ terms, these values are country-specific. Consequently, we have that $\lim _{\underline{T} \rightarrow \infty} \theta_{c}=0 \forall c \in$ $\{1,2, \ldots, C\}$ and $\theta_{0}=1$. Using an analogous argument, we also have $\lim _{\underline{T} \rightarrow \infty} \overline{\Delta y}_{c}=0$ and thus $\lim _{\underline{T} \rightarrow \infty} k=0$. Finally, combining these results with equations (9) and (6), we can conclude that $\lim _{\underline{T} \rightarrow \infty} \omega_{c}=0 \forall c \in C$ and $\lim _{\underline{T} \rightarrow \infty} \hat{\rho}=0$. 


\section{A.2 Replication of Figures and Tables using Penn World Tables}

Figure A-1: Empirical distribution of the underlying $\rho_{c}$ 's and $\omega_{c}$ 's

Panel A: Probability density of the $\rho_{c}$ 's

Panel B: Cumulative density of the $\rho_{c}$ 's

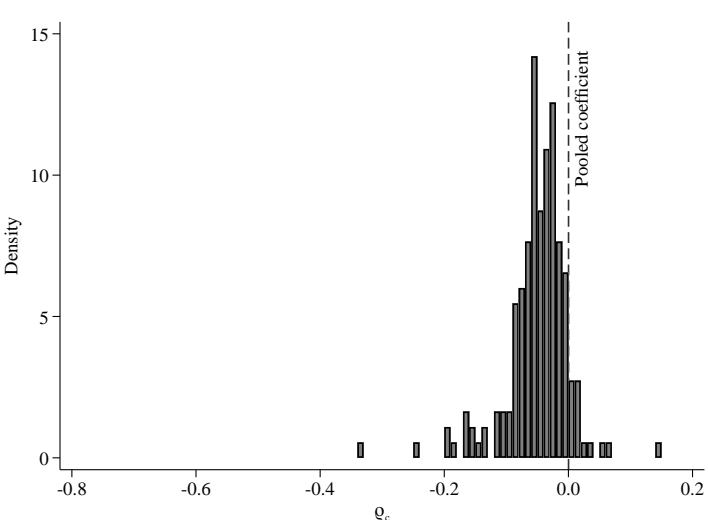

Panel C: Probability density of the $\omega_{c}$ 's

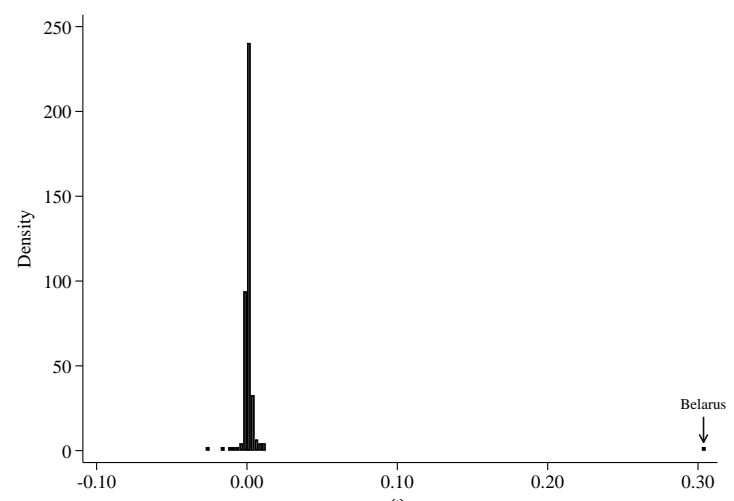

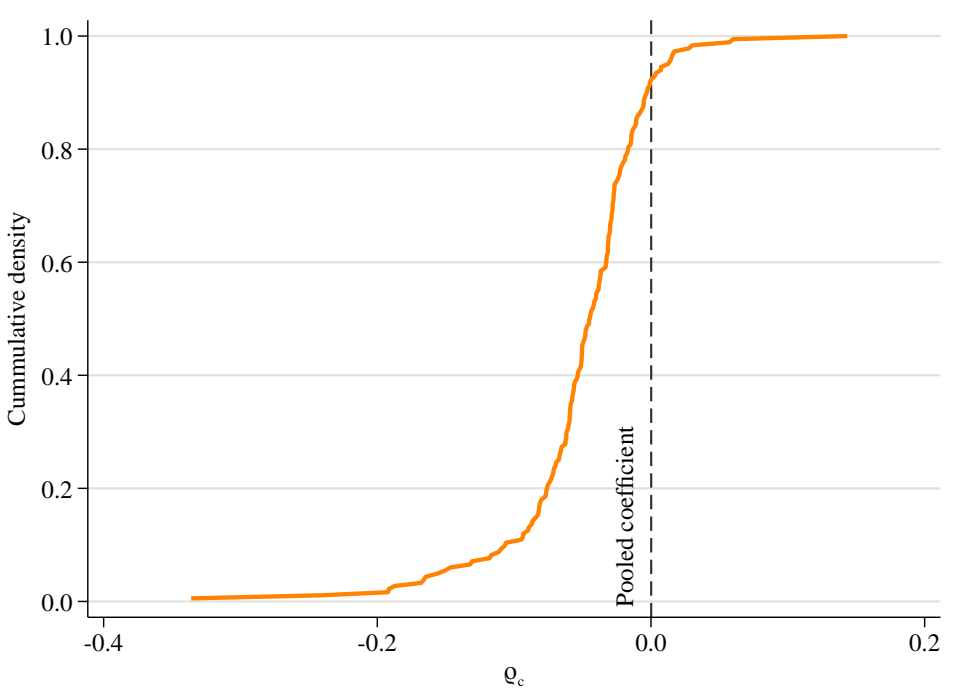

Panel D: Cumulative density of the $\omega_{c}$ 's

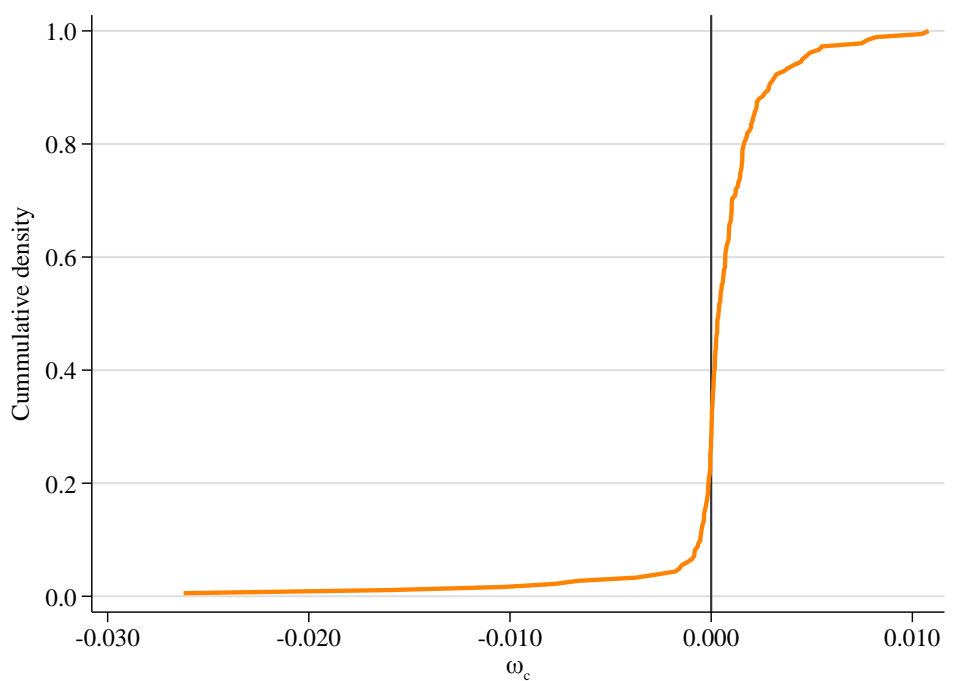

Notes: The figure presents estimates of the distribution of the country-specific coefficients of convergence (the $\rho_{c}$ 's), and the weights (the $\omega_{c}$ 's, defined in Proposition 1]. The empirical probability density and the cumulative density are reported on the left and the right-hand side panels respectively. In Panel A, the dashed line indicates the estimate $\hat{\rho}$ from equation 2 (when no country heterogeneity is allowed). 


\section{Figure A-2: Decomposition of $\hat{\rho}$ across decades}

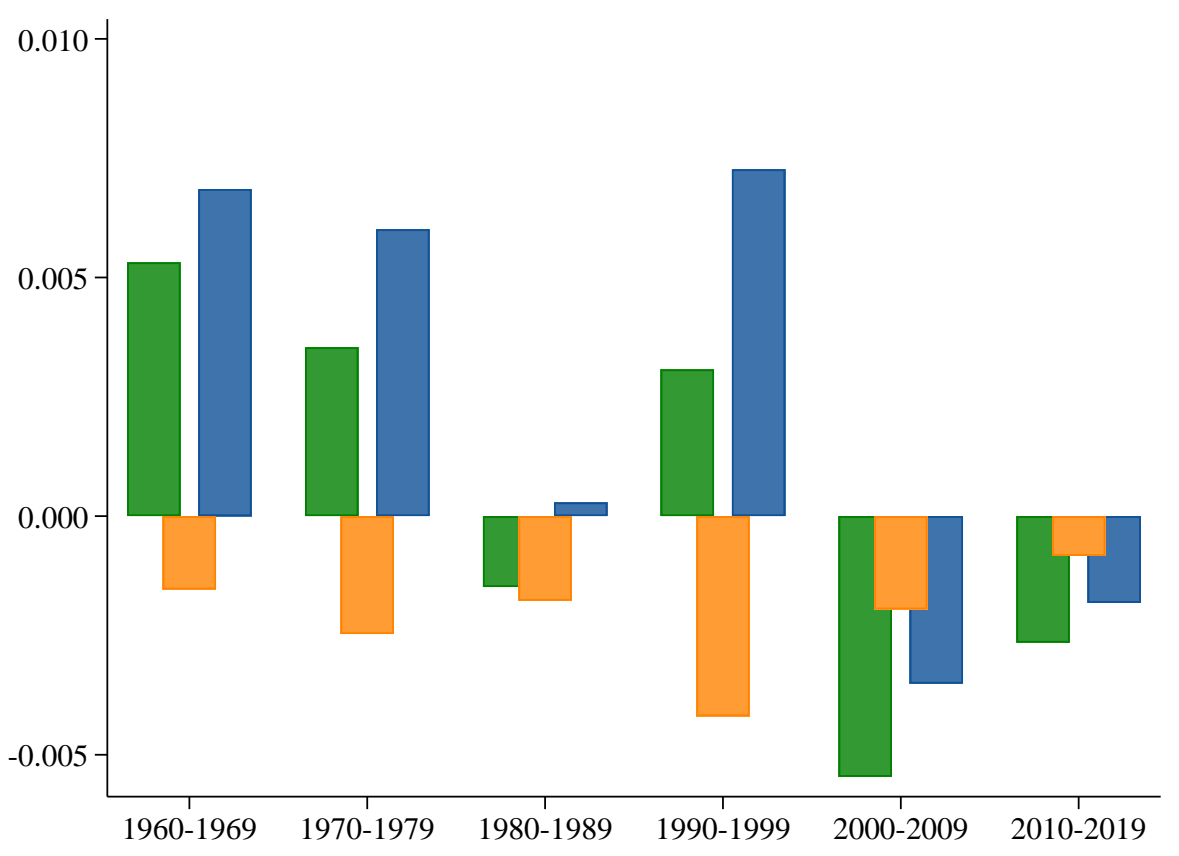

Notes: The figure reports the estimate $\hat{\rho}$ from equation 22 (when no country heterogeneity is allowed) by decade shown by the blue bar. This estimate is decomposed in two terms (see Proposition 1): the underlying distribution of the $\rho_{c}$ 's shown by the orange bar and the bias shown by the green bars. 
Figure A-3: Estimates of the relationship of Polity 2 and economic growth over time

Panel A: Dependent variable is 10-year changes

$\Delta_{10} y_{c t}=\beta_{t} x_{c t}+\rho_{t} y_{c t}+\delta_{t}+\varepsilon_{c t}$

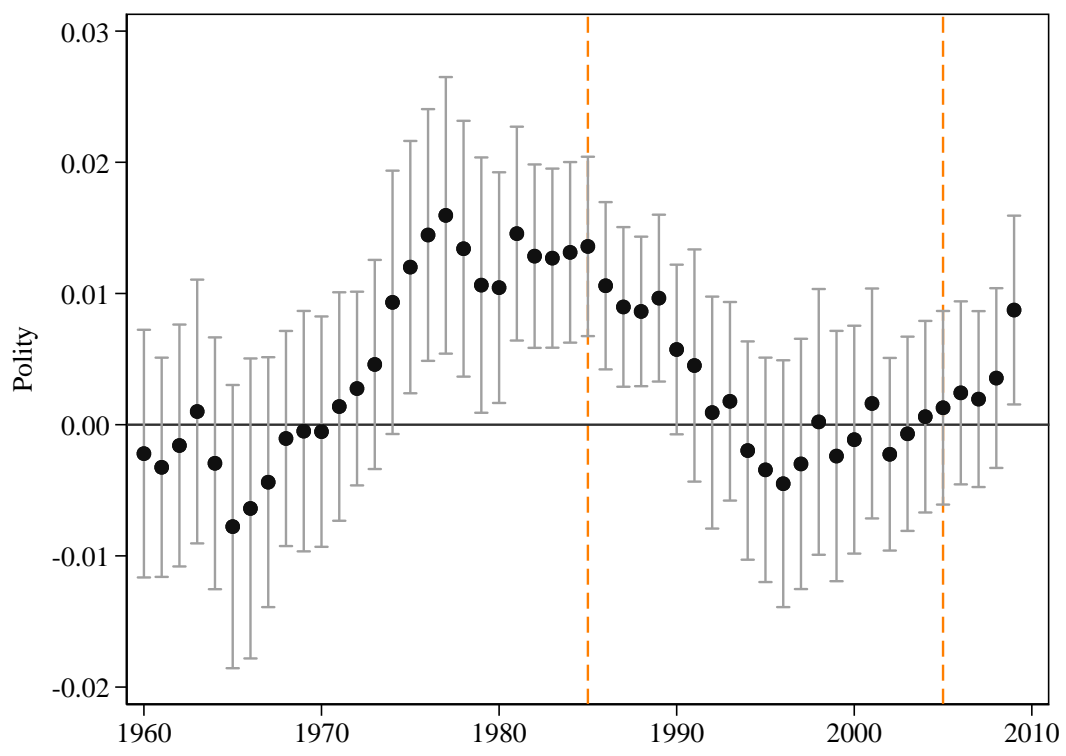

Panel B: Dependent variable is first difference

$\Delta y_{c t}=\beta_{t} x_{c t}+\rho_{t} y_{c t}+\delta_{t}+\varepsilon_{c t}$

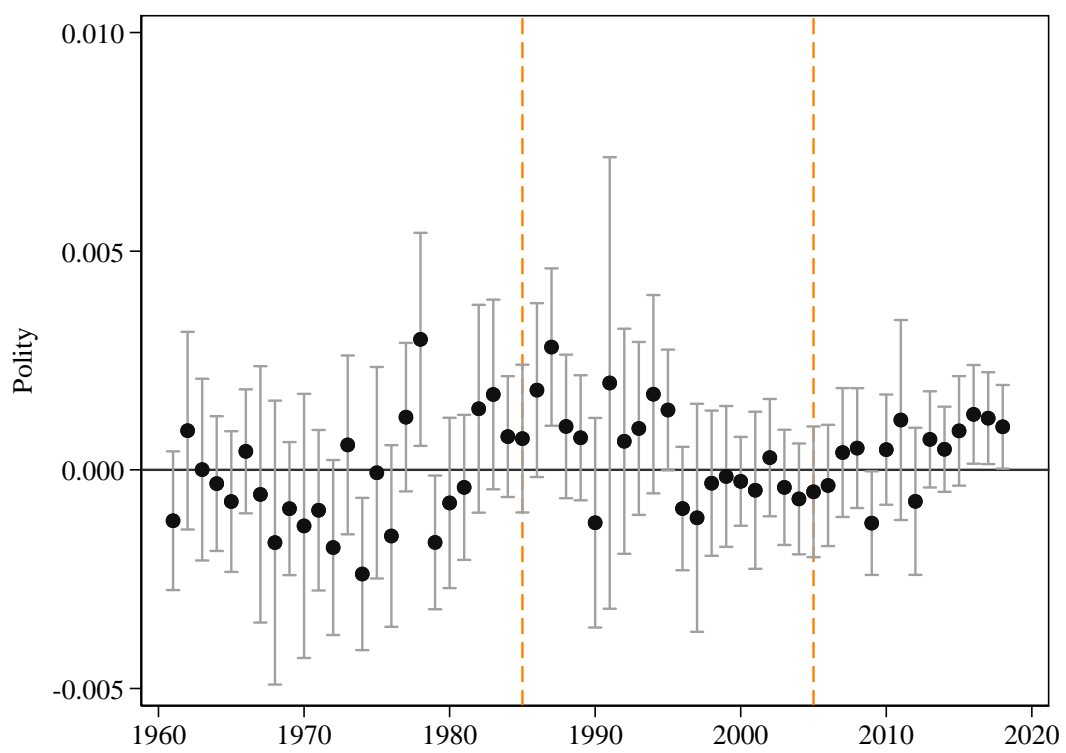

Notes: The figure reports estimates of the relationship between the Polity 2 score and economic growth and $95 \%$ confidence intervals over time, Namely, we plot the coefficient $\beta_{t}$ in the equation indicated in the panel labels. Panel A uses as dependent variable the 10-year change in log of GDP per capita, while Panel B uses annual changes (first differences). Standard errors are clustered at the country level. 


\section{Figure A-4: Estimates of $\rho$ across different specification and decades}

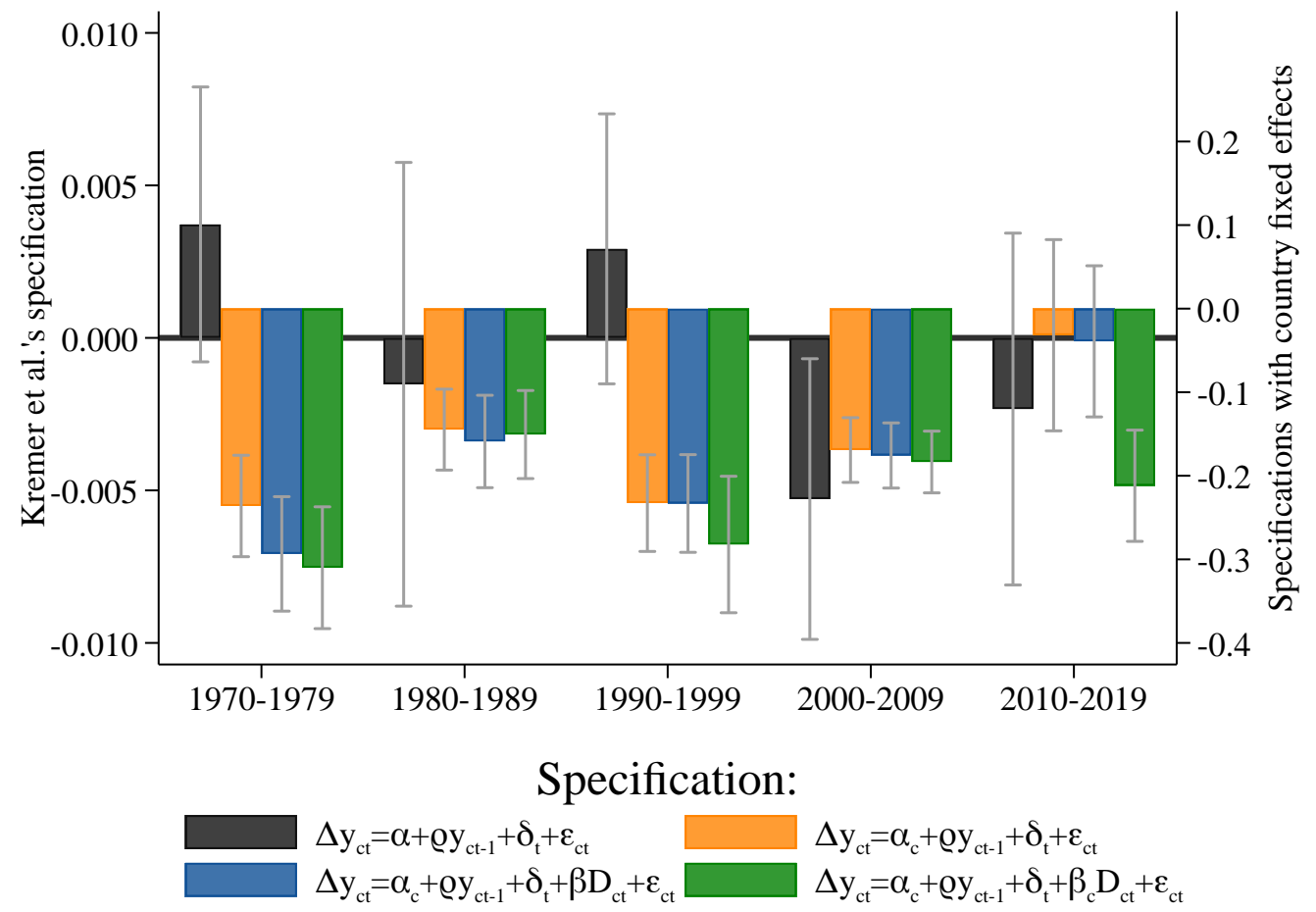

Notes: The figure report estimates of the coefficient of convergence $(\rho)$ as well as $95 \%$ confidence intervals across four different specifications. The first is Kremer et al. s specification of unconditional convergence, which does not include country fixed effects (shown by the black bars). The second is a specification that adds country fixed effects to this baseline (shown by the orange bars). The third adds our dichotomous measure of democracy as a control, focusing on the OLS specification (shown by blue bars). Our final specification allows the effects of democracy to be varying across countries green bars). Standard errors are clustered at the country level. 


\section{Figure A-5: Correlation of country fixed effects over decades}

Panel A: Main dependent is first difference, $\Delta y_{c t}=\rho y_{c t-1}+\delta_{t}+\alpha_{c, d}+\varepsilon_{c t}$
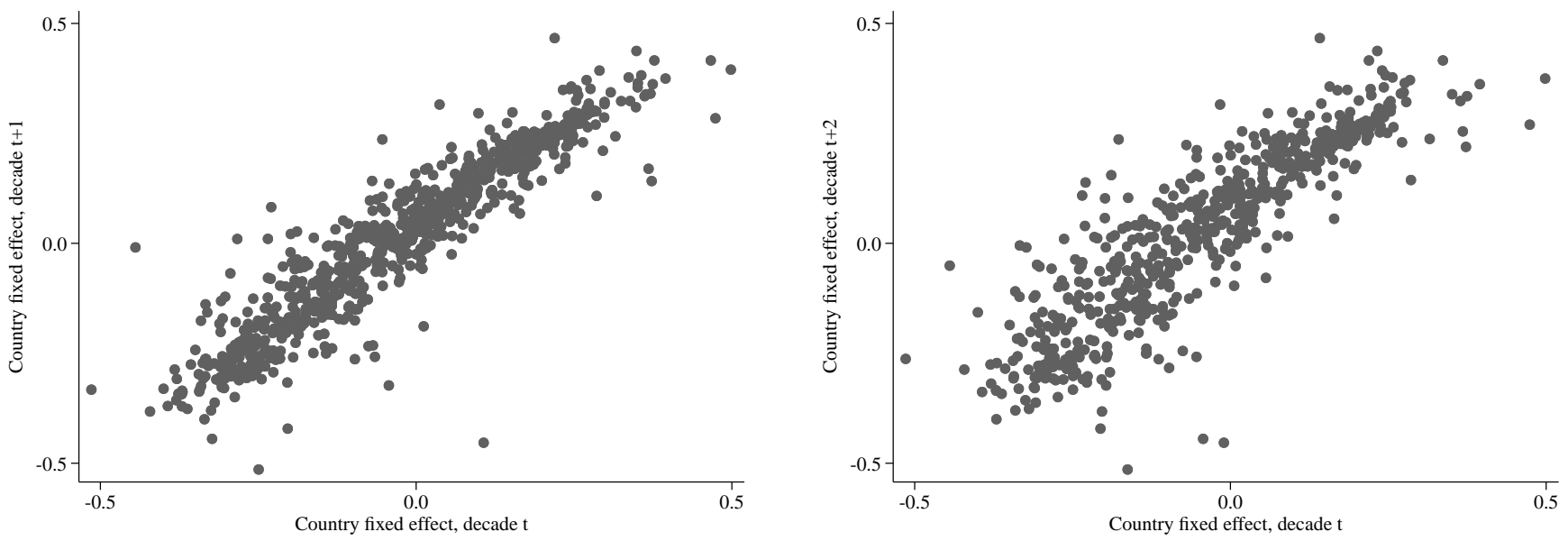

Panel B: Main dependent variable is 10-year changes, $y_{c, t+10}-y_{c, t}=\rho y_{c t}+\delta_{t}+\alpha_{c, d}+\varepsilon_{c t}$
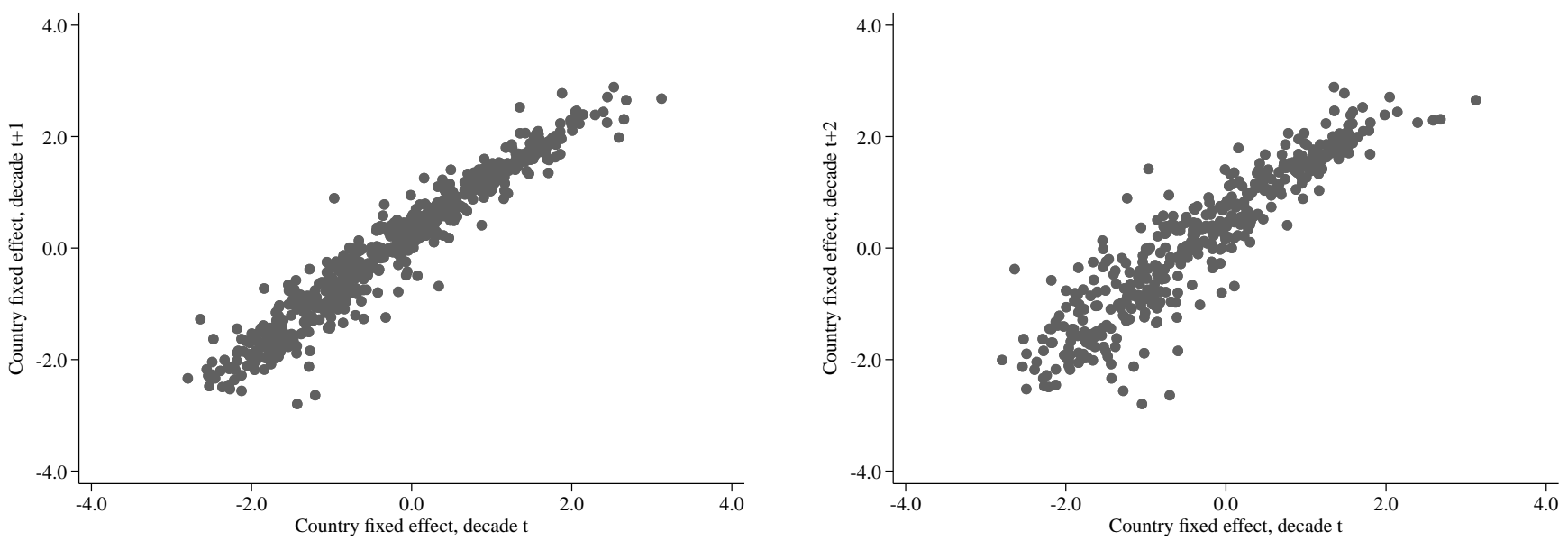

Notes: We estimate the regression specified in the panel label which allows the country fixed effects to vary across decades and then plot these decadal fixed effect estimates against each other. Panels A and B use as dependent variable the first difference (the 10-year change) in log of GDP per capita, while Panels C and D use 10-year changes. The panels to the left (right) compare shows the correlation between the estimated country fixed effect for a decade starting at year $\mathrm{t}$ with a decade starting at year $t+10(t+20)$. 
Table A-1: Convergence patterns for the key covariates

\begin{tabular}{lccccc}
\hline \hline & $(1)$ & $(2)$ & $(3)$ & $(4)$ & $(5)$ \\
& Pooled & \multicolumn{4}{c}{ Country-level coefficients } \\
\cline { 3 - 5 } & coefficient & Mean & 25th quantile & 50th quantile & 75th quantile \\
\hline \multirow{2}{*}{ Log GDP per capita } & 0.000 & -0.050 & -0.067 & -0.044 & -0.023 \\
Polity 2 & -0.029 & -0.107 & -0.165 & -0.087 & 0.000 \\
Rule of law & -0.008 & -0.346 & -0.487 & -0.295 & -0.169 \\
Property rights & -0.032 & -0.238 & -0.360 & -0.228 & -0.133 \\
Government expenditure & -0.055 & -0.215 & -0.313 & -0.168 & -0.069 \\
Credit & 0.004 & -0.221 & -0.403 & -0.114 & -0.013 \\
Years of schooling & -0.020 & -0.506 & -0.653 & -0.440 & -0.321 \\
\hline \hline
\end{tabular}

Notes: The table reports estimates of coefficient of unconditional convergence ( $\rho$, see equation $\sqrt{2}$ ) $)$ in column 1. The remaining columns report moments (including the mean and the 25th, 50th and 75th quantiles) of the distribution of the underlying country-specific estimates of convergence ( $\rho_{c}$ 's, see equation (10). We show results for the key variables used by Kremer et al. including (source in parenthesis): Polity 2 (Polity IV Project), Rule of law (Worldwide Governance Indicators), Property rights (Heritage Freedom), Government expenditure (World Development Indicators) and Years of schooling (Barro-Lee). 


\section{Table A-2: Estimates of the Effect of democracy on (log) GDP per capita}

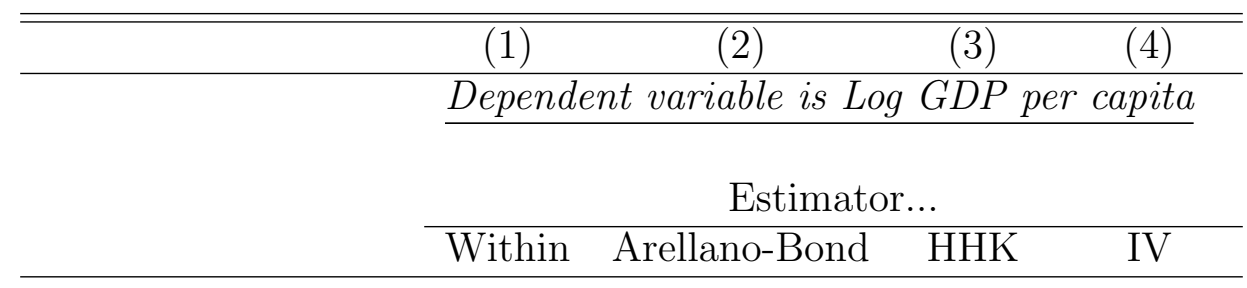

\section{A. Effect of Democracy on $\log G D P$}

\begin{tabular}{lcccc} 
Democracy & 0.009 & 0.014 & 0.005 & 0.005 \\
& $(0.004)$ & $(0.006)$ & $(0.007)$ & $(0.010)$ \\
Observations & 5,961 & 5,796 & 5,961 & 5,937 \\
\hline
\end{tabular}

B. Effect of Democracy on Log GDP and its change over time

\begin{tabular}{lcccc} 
Democracy & 0.009 & 0.014 & 0.004 & 0.006 \\
& $(0.004)$ & $(0.006)$ & $(0.006)$ & $(0.011)$ \\
Democracy $\times$ Trend & 0.000 & 0.004 & 0.001 & 0.002 \\
& $(0.003)$ & $(0.005)$ & $(0.004)$ & $(0.005)$ \\
Observations & 5,961 & 5,796 & 5,961 & 5,937 \\
\hline
\end{tabular}

Notes: This table presents estimates of the effect of democracy on log GDP per capita following Acemoglu et al. (2019). Democracy is measured as a dichotomous variable to minimize measurement error. Panel A replicates the results in Acemoglu et al. (2019), and Panel B extends the regression by allowing an interaction between the measure of democracy and a linear function of time. Column 1, 2, 3 and 4 present results from the within estimator, the Arellano and Bond/s (1991) GMM estimator, the HHK (Hahn et al. 2001) estimator, and an IV (exploiting regional waves of democratization) respectively. All regressions include four lags of log GDP per capita. Standard errors are clustered at the country level. 